\title{
Begriffliche Erkundung semantischer Strukturen von Sprechaktverben
}

Anja Großkopf, Gisela Harras

\section{Inhalt}

1. Klassifikationsprobleme von Sprechaktverben

2. Die Aufbereitung der semantischen Daten durch begriffliches Skalieren

3. Liniendiagramme und was sie zeigen

4. Möglichkeiten semantischer Erkundungen in TOSCANA

5. Ausblick

\section{Klassifikationsprobleme von Sprechaktverben}

\subsection{Eine Motivation für die TOSCANA-Anwendung: das Problem der Klassifikation von Sprechaktverben}

In diesem Beitrag soll eine TOSCANA-Anwendung für Sprachdaten, speziell Sprechaktverben, vorgestellt werden. Die Motivation für diese Anwendung ist im wesentlichen durch das linguistische Problem der Klassifikation dieses Wortschatzausschnitts begründet, dessen Bezugsbereich - grob umrissen - Akte des Kommunizierens oder technischer gesprochen: Sprechakte darstellen. Mit einer Klassifikation solcher Verben sollen zwei Zwecke verfolgt werden: zum einen sollen Bedeutungsrelationen, in denen die einzelnen Ausdrücke zueinander stehen, erfaßt werden und zum anderen soll damit gezeigt werden, welche Kommunikationskonzepte lexikalisch ausdrückbar sind und welche nicht. Klassifikationsmodelle finden sich bei Vertretern der philosophischen Sprechakttheoretiker, auf deren Vorschläge auch in linguistischen Arbeiten zur Semantik von Sprechaktverben zurückgegriffen wird.

Bereits der Begründer der Sprechakttheorie John L. Austin hat den Versuch unternommen, eine Klassifikation von Sprechakten vermittels derjenigen Verben, die sie bezeichnen, zu skizzieren. Als Kriterien führt er die "illocutionary force", also die kommunikative Sprecherabsicht, sowie die meist institutionenspezifische Situation an (vgl. [Au75]). Fortgeführt wurde dieser Klassifikationsversuch von John R. Searle, dessen Ergebnis einerseits als klassisch angesehen, zum andern aber auch heftig kritisiert wurde. Searle teilt die Sprechakte bekanntlich in fünf Kategorien ein, die durch vier Kriterien bestimmt sind (vgl. [Se80]): 


\begin{tabular}{|l||c|c|c|c|}
\hline & (i) & (ii) & (iii) & (iv) \\
\hline $\begin{array}{l}\text { Repräsentative } \\
\text { z. B. behaupten }\end{array}$ & $\vdash$ & $\downarrow$ & G & p \\
\hline $\begin{array}{l}\text { Direktive } \\
\text { z. B. bitten }\end{array}$ & ! & $\uparrow$ & W & H tut p \\
\hline $\begin{array}{l}\text { Kommissive } \\
\text { z. B. geloben }\end{array}$ & K & $\uparrow$ & A & S tut p \\
\hline $\begin{array}{l}\text { Expressive } \\
\text { z. B. fluchen }\end{array}$ & E & $\emptyset$ & (P) & S/H \& E \\
\hline $\begin{array}{l}\text { Deklarative } \\
\text { z. B. taufen }\end{array}$ & D & $\uparrow$ & $\emptyset$ & p \\
\hline
\end{tabular}

Abbildung 1 Die fünf Kategorien nach Searle

(i) das Kriterium des illokutionären Zwecks, der kommunikativen Sprecherabsicht, die durch die folgenden fünf Ausprägungen charakterisiert ist:

- das Erheben eines Wahrheitsanspruchs (Repräsentative), notiert als $\vdash$

- den Zweck der Direktive (Aufforderungen), notiert als !

- den Zweck der Kommissive (Verpflichtungen), notiert als K

- den Zweck der Expressive (Gefühlsäußerungen), notiert als E

- den Zweck der Deklarative (institutionsgebundene Äußerungen), notiert als D

(ii) das Kriterium der Ausrichtung:

- von der Welt zur Sprache (für Behauptungen), notiert als $\downarrow$

- von der Sprache zur Welt (für Aufforderungen und Verpflichtungen), notiert als $\uparrow$

- keine Ausrichtung (für expressive Äußerungen), notiert als $\emptyset$

- bidirektionale Ausrichtung (für deklarative Äußerungen wie Kriegserklärungen), notiert als $\downarrow$

(iii) das Kriterium der propositionalen Einstellung, d. h. der Einsstellung, die ein Sprecher zu dem hat, was er sagt:

- glauben $=\mathrm{G}$

- wollen $=\mathrm{W}$

- Absicht, das, was man sagt, zu tun = A

- emotional wertende Einstellung $=(P)$

- ohne $=\emptyset$

(iv) das Kriterium des propositionalen Gehalts als:

- beliebiger Sachverhalt p

- Handlung des Hörers: H tut $\mathbf{p}$

- Handlung des Sprechers: S tut p

- bestimmte Eigenschaften (Einstellungen, Gefühle) von S und H, notiert als: $\mathrm{S} / \mathrm{H} \& \mathrm{E}$ 
Insgesamt sind die Kriterien auf die fünf Kategorien wie folgt verteilt:

Gegen diese Klassifikation ist der Vorwurf erhoben worden, daß sie gegen das Prinzip der Disjunktivität, nach dem jedes zu klassifizierende Element nur in eine Kategorie fallen darf, verstößt (vgl. [Ba79]; [Me90]; [Ul92]). Die Kategorien der Klassifikation sind so konstituiert, daß die Zugehörigkeit eines Elements zu einer Kategorie seine Zugehörigkeit zu einer anderen Kategorie impliziert. Dafür sind vor allem zwei Begründungen angeführt worden:

(1) Direktive sind immer auch Repräsentative

(2) Kommissive sind immer auch Repräsentative

Für die erste Begründung wird folgende sprechakttheoretische Überlegung angeführt: ein illokutionäre Zweck jeder Äußerung ist das Verstandenwerden durch den Hörer, d.h. das Zutreffen bestimmter Glaubenszustände, im Fall von Direktiven wäre dies:

$$
\mathrm{S} \text { will, daß H glaubt, daß p (mit } \mathrm{p}=\text { „S will, daß H r tut") }
$$

Die Beschreibung (1.1) ist äquivalent mit der von Repräsentativen aus der Searleschen Klassifikation. Mit ihr ist festgehalten, daß Akte wie Auffordern, Bitten, Anordnen, Anweisen usw. auch immer Informationsakte implizieren, oder verbsemantisch formuliert, daß zu den Bedeutungsimplikationen von auffordern, bitten, anordnen, anweisen usw. auch „mitteilen, daß S will, daß $\mathrm{H} \mathrm{r}$ tun soll“, gehört.

Zur zweiten Begründung: Kommissive sind auch Repräsentative, insofern sie dem Hörer eine bestimmte Sprecherabsicht mitteilen:

(1.2) $\mathrm{S}$ will, daß H glaubt, daß $\mathrm{p}$ (mit $\mathrm{p}=$ „S will $\mathrm{r}$ tun“)

Aus den beiden Beispielen geht hervor, daß man das Problem der Klassifikation von Sprechakten bzw. den Verben, die sie bezeichnen, am besten durch die Berücksichtigung der Möglichkeit mehrdimensionaler hierarchischer Klassifikationen lösen kann. In den beiden genannten Fällen heißt dies: unter dem Aspekt des illokutionären Zwecks des Verstandenwerdens allein ( $H$ glaubt p) sind Direktive und Kommissive äquivalent mit Repräsentativen oder verbsemantisch formuliert: Verben wie auffordern, bitten, sich verpflichten, versprechen implizieren "mitteilen, daß $\mathrm{p}^{\prime}$. Unter dem Aspekt der speziellen Ausprägung der jeweiligen propositionalen Gehalte fallen auffordern (mit $\mathrm{p}={ } \mathrm{H}$ tut $\mathrm{r}^{\prime \prime}$ ), versprechen (mit $\mathrm{p}=, \mathrm{S}$ tut $\mathrm{r}^{\prime)}$ ) und mitteilen (mit unspezifischem $p$ ) in je unterschiedliche Kategorien.

Es versteht sich von selbst, daß Mehrfachzuordnungen zu unterschiedlichen Kategorien eher den Normalfall als die Ausnahme darstellen; mit den beiden Beispielen sollte lediglich gezeigt werden, daß sich das Problem bereits bei der Anwendung der fundamentalen Klassifikationskriterien stellt.

Angesichts dieser Sachlage erscheint es uns vielversprechend, eine TOSCANA-Anwendung für die Klassifikation von Sprechaktverben auszuprobieren, vor allem um damit folgendes transparent zu machen: 
- Die Zugehörigkeit eines Elements der Klassifikation, d. h. eines bestimmten Verbs, ist abhängig von der jeweiligen Kombination und Gewichtung der einzelnen Kriterien.

- Die Kombination und Gewichtung der einzelnen Kriterien ist abhängig von dem Bedürfnis, d.h. dem Erkenntnisinteresse der einzelnen Benützer, in diesem Fall von Linguisten, die etwas über die Semantik von Sprechaktverben herausfinden wollen.

- Die Klassifikation von Sprechaktverben muß nicht notwendigerweise dem Prinzip der Saturiertheit genügen, nach dem jede Kategorie mindestens ein Element enthalten muß. Nicht saturierte Klassifikationen lassen Voraussagen zu (vgl. [U192]), die durch TOSCANA stipuliert werden können.

\subsection{Der semantische Bezugsrahmen für Sprechaktverben}

Im folgenden geben wir einen Überblick über die Komponenten, die insgesamt den semantischen Bezugsrahmen für die Klassifikationsmöglichkeiten der einzelnen Verben darstellen. Der hier zugrunde gelegte Ausschnitt des Verbwortschatzes ist durch vier invariante Merkmale gekennzeichnet, die den Typ von Situationen charakterisieren, auf den mit jedem Verb Bezug genommen wird, z. B. für das Verb versprechen in:

(1) Franz versprach Fritz, beim Umzug zu helfen.

Die Merkmale sind die folgenden:

1. Es gibt eine Sprecher $\mathbf{S}(\mathbf{S}=$ Franz)

2. Es gibt einen Hörer (Adressaten) $\mathrm{H}(\mathrm{H}=$ Fritz)

3. Es gibt ein Äußerungsprodukt von $\mathrm{S}$ einem $\mathrm{H}$ gegenüber mit einem propositionalen Gehalt $p(p=$,beim Umzug helfen“)

4. Es gibt eine kommunikative Einstellung von $S$ („Verpflichtung, beim Umzug zu helfen")

Diese vier Merkmale konstituieren einen allgemeinen Typ von Bezugssituation als konzeptuelle Invariante der Verwendung aller betrachteten Verben.

Die Merkmale 3 und 4 können weiter differenziert werden, um spezielle Typen von Bezugssituationen der Verben zu unterscheiden. Die theoretische Basis der Differenzierung liefert eine sprechakttheoretisch fundierte Semantik, wie sie z. B. in [Bau77]), [Se85]), [Va90]) vorgeschlagen wurde.

Für das Merkmal 3 - Äußerungsprodukt von $\mathrm{S}$ einem $\mathrm{H}$ gegenüber mit einem propositionalen Gehalt - bezieht sich die Differenzierung auf folgende Eigenschaften propositionaler Gehalte:

- Gehalt: Mitteilungsgehalt - Fragegehalt

- Geschehenstyp

- Rollenbezug

- Zeitbezug 
Diese Eigenschaften haben die folgenden Merkmale (Ausprägungen); für „Geschehenstyp":

- Zustand

- Ereignis

- Handlung

wobei das Merkmal „Handlung“ eine Spezialisierung des Merkmals „Ereignis" darstellt. Linguistisch gesehen gestattet diese Unterscheidung eine erste semantische Strukturierung von Verben wie behaupten, erklären, mitteilen einerseits und Verben wie sich verpflichten, anflehen, raten, vorschlagen andererseits und erklärt die semantische Anomalie von Sätzen wie:

(2) * Er verpflichtet sich zur Wetterlage

(3) * Sie schlug vor, daß die Bäume blühn

(4) * Er fleht sie an, die Sonne zu scheinen usw.

(Die * markieren die semantische Abweichung.)

Für „Rollenbezug“, d.h. die Ausprägung des Trägers eines Zustands oder einer Handlung, gelten die folgenden Merkmale:

- Sprecher: S

- Hörer: H

- Sprecher und Hörer: S und H

- Dritte

- H oder Dritte

Dies gestattet semantische Differenzierungen zwischen versprechen (Rollenbezug: S), bitten (Rollenbezug: $\mathrm{H}$ ), vorschlagen (Rollenbezug: $\mathrm{S}$ und $\mathrm{H}$ ) oder festsetzen (Rollenbezug: H oder Dritte).

Für „Zeitbezug“ gelten die Merkmale:

- vergangen: prät

- gegenwärtig: präs

- zukünftig: fut

Distinktiv sind hier lediglich die Merkmale prät und fut; vgl. bereuen, beklagen, verurteilen vs. ankündigen, geloben, bitten.

Alle Verben des charakterisierten allgemeinen Typs von Bezugssituation lassen sich bezüglich dieser Merkmale charakterisieren, d.h. ihnen können bestimmte Kombinationen solcher Merkmale zugewiesen werden; z. B. hat dann das Verb bereuen die folgende Merkmalskombination:

- Geschehenstyp: Handlung

- Rollenbezug: S

- Zeitbezug: prät 


\begin{tabular}{|c|c|c|c|}
\hline Merkmale & \multirow[b]{2}{*}{ Geschehenstyp } & \multirow[b]{2}{*}{ Rollenbezug } & \multirow[b]{2}{*}{ Zeitbezug } \\
\hline Gegenstände Auspr. & & & \\
\hline bereuen & Handlung & $S$ & prät \\
\hline vorschlagen & Handlung & $S$ und $H$ & fut \\
\hline zusichern & Handlung & $S$ & fut \\
\hline bitten & Handlung & $\bar{H}$ & fut \\
\hline taufen & Zustand & H und Dritte & fut \\
\hline
\end{tabular}

Abbildung 2 Charakterisierung von Verben bzgl. der Bezugssituation

Und das Verb auffordern:

- Geschehenstyp: Handlung

- Rollenbzug: $\mathrm{H}$

- Zeitbezug: fut

Das Verb versprechen

- Geschehenstyp: Handlung

- Rollenbzug: S

- Zeitbezug: fut

Diese Verteilung kann man auch in Form von Tabellen darstellen, wobei die Zeilen die Gegenstände, d.h. in diesem Fall die Verben, verzeichnen und die Spalten die Merkmale, vgl. Abb. 2.

Diese Tabelle stellt einen mehrwertigen Kontext dar, in dem Gegenständen bestimmte Merkmalsausprägungen zugeordnet sind. Der Kontext ist ein Welt- bzw. in diesem speziellen Fall ein Sprachausschnitt. Eine traditionelle linguistische Analyse der Sprachdaten wäre nun auf die Ausarbeitung der vollständigen Tabelle zur Merkmalsverteilung aller zugrundegelegter Verben angewiesen, um Aussagen über die jeweiligen Bedeutungen der einzelnen Verben im Zusammenhang mit allen anderen Verben des Paradigmas zu ermöglichen. Mit Paradigma oder Lexikalischem Feld wird eine Menge von Verben bezeichnet, die bezüglich eines oder mehrerer Merkmale übereinstimmen, also Begriffsumfänge im skalierten Kontext (vgl. Abschnitt 2 und drei) sind. Die Darstellung solcher Zusammenhänge würde sich als sehr komplex und unübersichtlich erweisen, zumal wir ja bisher nur die Eigenschaften propositionaler Gehalte und deren mögliche Ausprägungen betrachtet haben und noch nicht auf die sehr viel komplexeren Eigenschaften der kommunikativen Einstellung von $\mathrm{S}$ eingegangen sind. Um eine übersichtlichere und für den $\mathrm{Be}-$ nutzer/Leser nachvollziehbarere Darstellung der Datenstrukturen zu gewährleisten, wurde versucht, die Strukturen der Verben in einem TOSCANASystem zu repräsentieren und so eine systematische Erkundung der konzeptuellen Bedeutung der Verben zu ermöglichen. 


\section{Die Aufbereitung der semantischen Daten durch begriffliches Skalieren}

Die Aufbereitung der Daten geschieht mit Hilfe der Formalen Begriffsanalyse. Die Basisstruktur der Analyse ist der formale Kontext. Dieser besteht aus zwei Mengen, einer Gegenstandsmenge und einer Merkmalsmenge, sowie einer binären Relation zwischen den beiden Mengen. Üblicherweise wird ein Kontext durch eine Kreuztabelle dargestellt: Die Zeilen werden mit den Namen der Gegenstände beschriftet und die Spalten mit den Namen der Merkmale. Die Relation zwischen Gegenständen und Merkmalen wird dadurch dargestellt, daß man für jedes Paar $(g, m)$, welches Element der Relation ist, ein Kreuz an die entsprechende Stelle in der Tabelle setzt, d. h. genau da, wo sich die mit $g$ beschriftete Zeile und die mit $m$ beschriftete Spalte überschneiden. Man sagt dann: Der Gegenstand $g$ hat das Merkmal $m$. Im Rahmen der Theorie der Formalen Begriffsanalyse gibt es nun eine Methode, wie man von dieser Tabelle ausgehend zu einem Begriffsverband gelangt, der als Liniendiagramm dargestellt wird. Das entsprechende Liniendiagramm enthält in übersichtlicher Weise die gleiche Information wie der formale Kontext.

In unserem Fall stellt die Menge der Sprechaktverben die Gegenstandsmenge dar und die Menge der Eigenschaften, die die Bezugssituationen charakterisieren, die Merkmalsmenge. Diese Eigenschaften stehen als Elemente der Merkmalsmenge gleichberechtigt nebeneinander, die Kontextdarstellung der Formale Begriffsanalyse berücksichtigt zunächst nicht die hierarchische Beziehung zwischen Eigenschaften (vgl. dazu Abschnitt 5).

Wie in Abb. 2 ersichtlich wird, hat jedes Verb bezüglich jeder Eigenschaft eine Ausprägung, die sich nicht einfach durch "hat die Eigenschaft" bzw. "hat die Eigenschaft nicht" beschreiben läßt. Die jeweiligen Eigenschaftsausprägungen sind differenzierender Natur, d.h. in den Feldern der Tabelle stehen nicht Kreuze, sondern eben die Ausprägungen, die die Verben bei den entsprechenden Eigenschaften haben.

Um diesen mehrwertigen Kontext durch ein übersichtliches Liniendiagramm darstellen zu können, muß er in einen formalen Kontext transformiert werden. Dies geschieht durch begriffliches Skalieren, wodurch aus einem mehrwertigen Kontext ein (einwertiger) formaler Kontext wird. Die Gegenstände bleiben dieselben, d.h. der abgeleitete einwertige Kontext hat die gleiche Gegenstandsmenge wie der ursprüngliche mehrwertige, und jedes mehrwertige Merkmal wird durch ein oder mehrere einwertige Merkmale ersetzt. Auf welche Weise eine bestimmte Merkmalsausprägung, die ein $\mathrm{Ge}-$ genstand bezüglich eines Merkmals hat, im abgeleiteten einwertigen Kontext dargestellt wird, ist durch begriffliche Skalen vorgeschrieben. Diese geben die Transformationsregeln für jeweils ein Merkmal an. Eine begriffliche Skala eines mehrwertigen Merkmals wird durch einen formalen Kontext repräsentiert: Die Gegenstandsmenge ist die Menge der möglichen Ausprägungen des mehrwertigen Merkmals, und die Merkmalsmenge ist die Menge der einwertigen Merkmale, durch die das Merkmal im abgeleiteten Kontext ersetzt werden 


\begin{tabular}{|c|c|c|c|c|c|c|c|c|}
\hline & 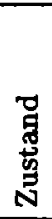 & 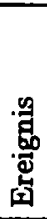 & $\begin{array}{l}\text { 骂 } \\
\text { 㝵 } \\
\text { 基 }\end{array}$ & n & 江 & $\begin{array}{l}1 \\
2 \\
\text { os }\end{array}$ & 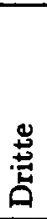 & 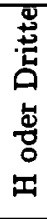 \\
\hline Zustand & $\bar{x}$ & & & & & & & \\
\hline Ereignis & & $x$ & & & & & & \\
\hline Handlung & & $x$ & $x$ & & & & & \\
\hline Zustand, S & $x$ & & & $x$ & & & & \\
\hline Zustand, $\mathrm{H}$ & $x$ & & & & $x$ & & & $x$ \\
\hline Zustand, S \& H & $x$ & & & $x$ & $x$ & $x$ & & $x$ \\
\hline Zustand, Dritte & $x$ & & & & & & $x$ & $x$ \\
\hline Zustand, $\mathrm{H}$ oder Dritte & $x$ & & & & & & & $x$ \\
\hline Handlung, $S$ & & $x$ & $x$ & $x$ & & & & \\
\hline Handlung, $\mathrm{H}$ & & $x$ & $x$ & & $x$ & & & $x$ \\
\hline Handlung, S \& H & & $x$ & $x$ & $x$ & $x$ & $x$ & & $x$ \\
\hline Handlung, Dritte & & $x$ & $x$ & & & & $x$ & $x$ \\
\hline Handlung, $\mathrm{H}$ oder Dritte & & $x$ & $x$ & & & & & $x$ \\
\hline$S$ & & & & $x$ & & & & \\
\hline $\mathbf{H}$ & & & & & $x$ & & & $x$ \\
\hline S\& H & & & & $x$ & $x$ & $x$ & & $x$ \\
\hline Dritte & & & & & & & $x$ & $x$ \\
\hline H oder Dritte & & & & & & & & $x$ \\
\hline
\end{tabular}

Abbildung 3 Skala für das Merkmal „Propositionaler Gehalt"

soll. Durch die Skalierung des mehrwertigen Kontextes wird die Ausprägung, die ein Gegenstand bezüglich eines Merkmals hat, durch das Kreuzchenmuster ersetzt, das diese Ausprägung als Gegenstand in der entsprechenden begrifflichen Skala als Inhalt hat. In Abb. 3 ist der abgeleitete formale Kontext der Merkmalsausprägung des propositionalen Gehalts wiedergegeben (vgl. Abb. 2).

Für jedes Merkmal wird eine begriffliche Skala benötigt. Die Skalen müssen weder alle identisch noch alle voneinander verschieden sein. Insbesonders gilt, daß es keine feste Vorgehensweise für die Erstellung von Skalen gibt. Vielmehr kann jeder beliebige formale Kontext als Skala herangezogen werden, wenn das sinnvoll ist. Welche Skala im einzelnen zum Skalieren eines mehrwertigen Kontextes zugrunde gelegt wird, muß hinsichtlich der Art der Daten, des Themengebiets und des Erkenntnisinteresses der jeweiligen Untersuchung, in diesem Fall also der Frage nach der Realisierung der konzeptuellen Merkmale als semantischer Gehalt der Sprechaktverben, festgelegt werden. 


\section{Liniendiagramme und was sie zeigen}

Durch formale Kontexte werden Begriffsverbände konstituiert. Ein formaler Begriff eines formalen Kontexts setzt sich aus einem Umfang und einem Inhalt zusammen, wobei der Umfang aus einer Menge von Gegenständen und der Inhalt aus einer Menge von Merkmalen besteht. Sie hängen insofern zusammen, als der Inhalt eines Begriffs die Menge all der Merkmale ist, die allen Gegenständen des Umfangs gemeinsam sind, und genauso ist der Umfang des Begriffs die Menge all derjenigen Gegenstände, die alle Merkmale des Inhalts haben. Ein Begriff ist durch eine der beiden Mengen, also Umfang oder Inhalt, schon vollständig definiert, da sich die eine aus der jeweils anderen ergibt. Alle Begriffe eines formalen Kontexts bilden - mit der Mengeninklusion bezüglich der Umfänge als Ordnung - einen Verband: Das ist der Begriffsverband des formalen Kontexts. Solche Verbände werden durch Liniendiagramme dargestellt, die mit den Merkmalen und Gegenständen beschriftet sind. (Bei langen Gegenstandslisten oder auch der Übersicht halber mag es zuweilen opportun erscheinen, die Gegenstandslisten wegzulassen; stattdessen kann man sich dann statt dieser die Anzahl der Gegenstände angeben lassen.) Dabei wird der kleinste Begriff, der einen bestimmten Gegenstand im Umfang hat, mit dessen Gegenstandsnamen beschriftet; dieser Begriff wird auch als Gegenstandsbegriff des entsprechenden Gegenstands bezeichnet. Analog dazu wird für jedes Merkmal der größte Begriff, der dieses Merkmal im Inhalt hat, mit dessen Namen beschriftet; hier sprechen wir entsprechend vom Merkmalbegriff. Nehmen wir als Beispiel den Kontext, der entsteht, wenn man alle Sprechaktverben bezüglich Geschehenstyp, Rollenbezug und Zeitbezug charakterisiert; wir haben also die Sprechaktverben als Gegenstandsmenge und die Merkmale Geschehenstyp, Rollenbezug und Zeitbezug als Merkmalsmenge (Abb. 2 zeigt einen kleinen Ausschnitt dieses Kontextes). Dieser Kontext wird nun skaliert wie im vorigen Abschnitt beschrieben, das heißt also in einen formalen Kontext umgewandelt. Dazu verwenden wir die Skala von Abb. 3. Zu beachten ist dabei noch, daß nur die beiden ersten Spalten des Kontextes, also die Merkmale Geschehenstyp und Rollenbezug, beim Skalieren berücksichtigt werden; die zum Merkmal Zeitbezug gehörige dritte Spalte wird aus Gründen der Übersichtlichkeit vernachlässigt. In Abb. 4 ist das Liniendiagramm des durch diese Skalierung entstehenden Kontextes zu sehen.

Für Liniendiagramme gelten die folgenden Leseregeln: Ein Gegenstand hat ein Merkmal genau dann, wenn dessen Merkmalbegriff vom entsprechenden Gegenstandsbegriff aus durch einen aufsteigenden Linienzug im Liniendiagramm zu erreichen ist. An dem Liniendiagramm in Abb. 4 ist für die bearbeitete Datenmenge z. B. ablesbar, daß es 113 Verben gibt mit dem Merk-

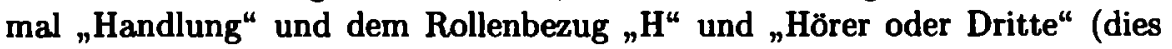
sind alle Aufforderungsverben), 17 Verben, nämlich beanstanden, bemängeln, kritisieren, kritteln usw. mit dem Merkmal "Handlung“ und dem Rollenbezug "H oder Dritte“ (dies sind alle Beanstandungsverben), und drei Verben, nämlich aufnehmen, initiieren und taufen mit dem Merkmal „Zustand" und 


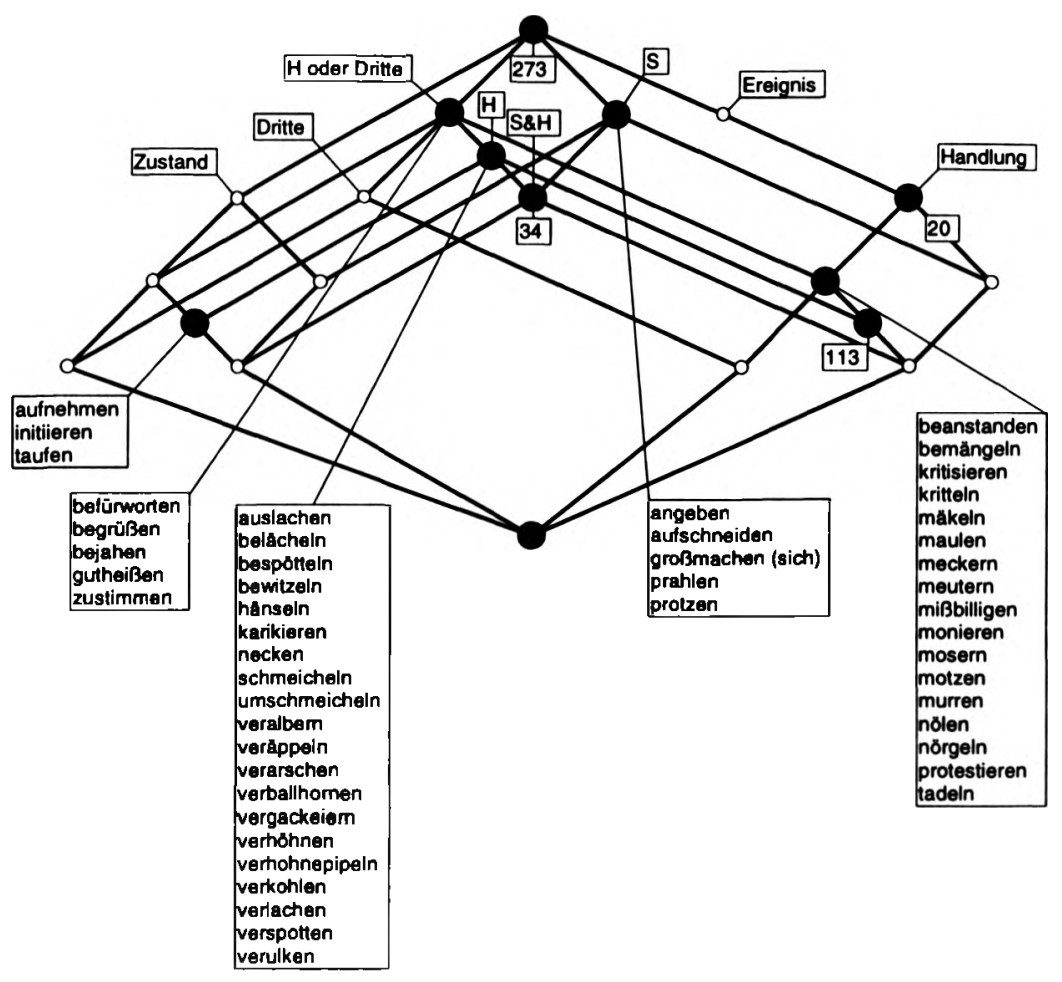

Abbildung 4 Liniendiagramm: Mitteilungsgehalt (propositionaler Gehalt)

dem Rollenbezug „H“ (dies sind institutionengebundene Verben). Einige der Punkte im Diagramm sind klein und nicht ausgefüllt; das bedeutet, daß im Umfang des zugehörigen Begriffes keine Merkmale sind, die nicht auch schon im Umfang eines echten Unterbegriffes zu finden sind. Wir sprechen auch von einem nichtrealisierten Begriff. Die großen ausgefüllten Punkte stehen für die sogenannten realisierten Begriffe, d. h. in ihrem Umfang gibt es Gegenstände, die nicht im Umfang eines echten Unterbegriffes liegen.

An diesem Liniendiagramm ist auch bereits ablesbar, was am Anfang dieses Beitrags zur Klassifikation von Sprechaktverben gesagt wurde: Unter einem Aspekt allein ergeben sich Kategorien mit zunächst sehr heterogenen Elementen, und die einzelnen Kategorien selbst erscheinen prima facie als äußerst divergierend wie z. B. die Kategorie der institutionengebundenen und die der Beanstandungsverben. 
Für ein weiteres aufschlußreiches Liniendiagramm untersuchen wir das vierte und letzte der anfangs aufgelisteten Merkmale, die den allgemeinen Typ der Bezugssituation charakterisieren, nämlich die kommunikative Einstellung von S. Diese ist auf der Basis der zugrundegelegten Theorie zu differenzieren in:

1. Propositionale Einstellung, d.h. einer Einstellung zu dem was man sagt, ob man es glaubt, will, für gut oder schlecht hält usw.

2. Intentionale Einstellung, d.h. einer Sprecherabsicht einem Hörer gegenüber

3. Situative Vorannahme von $\mathrm{S}$ aus seiner Sicht

(1) „Propositionale Einstellung“ hat die folgenden systematisch möglichen Eigenschaften:

- „epistemische Einstellung“, d.h. einer Glaubens- oder Wissenseinstellung des Sprechers zu dem, was er sagt, z. B. ob er es für wahr oder nicht (vgl. behaupten vs. lügen)

- "voluntative Einstellung“, d.h. einer willentlichen Einstellung des Sprechers, ob er das, was er sagt (wozu er auffordert, rät usw.), will oder nicht (vgl. flehen vs. verbieten oder warnen)

- eine „ordinative Einstellung“, d.h. eine Einstellung des Einordnens eines Gegenstands/Sachverhalts (vgl. $X$ als $Y$ beurteilen, einstufen)

- eine „evaluative Einstellung“, d. h. eine wertende Einstellung des Gesagten, die Einschätzung als gut oder schlecht (vgl. kritisieren, bereuen, loben)

- eine „emotive Einstellung“, d. h. eine emotionale Einstellung zum Gesagten wie Leid, Ärger und Freude (vgl. klagen, jubeln, nörgeln)

Für jede dieser Eigenschaften gibt es Liniendiagramme, die den Begriffsverband der jeweiligen Ausprägungen veranschaulichen. Aus Platzgründen sei hier nur das Beispiel des Diagramms für „propositionale epistemische Einstellung" angeführt; vgl. Abb. 5.

Aus diesem Diagramm ist ablesbar, daß die Verben anflunkern, anlügen, anschwindeln, beflunkern etc. die Merkmale "nicht für wahr halten“ und " ${ }^{\text {“ }}$ haben (das sind alle Lügen-Verben), die Verben (beweisen), argumentieren, beharren, behaupten etc. die Merkmalen „für wahr halten" und „p" (das sind alle Verben des Behauptens und Bejahens) und die Verben abstreiten, $a b$ weisen, bestreiten, bezweifeln etc. die Merkmale „für wahr halten" und „ $\neg \mathrm{p}^{\text {" }}$ (das sind alle Verneinens-Bestreitensverben). Aus dem Diagramm ist - und dies ist für linguistische Zwecke ein nicht zu unterschätzender Wert - aber auch ablesbar, daß es für bestimmte Merkmalsausprägungen keine Verben im Deutschen gibt, z. B. für "nicht für wahr halten "־ p" oder „nicht kennen" "p“, denn bei den Begriffen, die genau diese Merkmale als Inhalt haben, handelt es sich um nichtrealisierte Begriffe.

(2) „Intentionale Einstellung“ hat die folgenden systematisch möglichen Eigenschaften: 


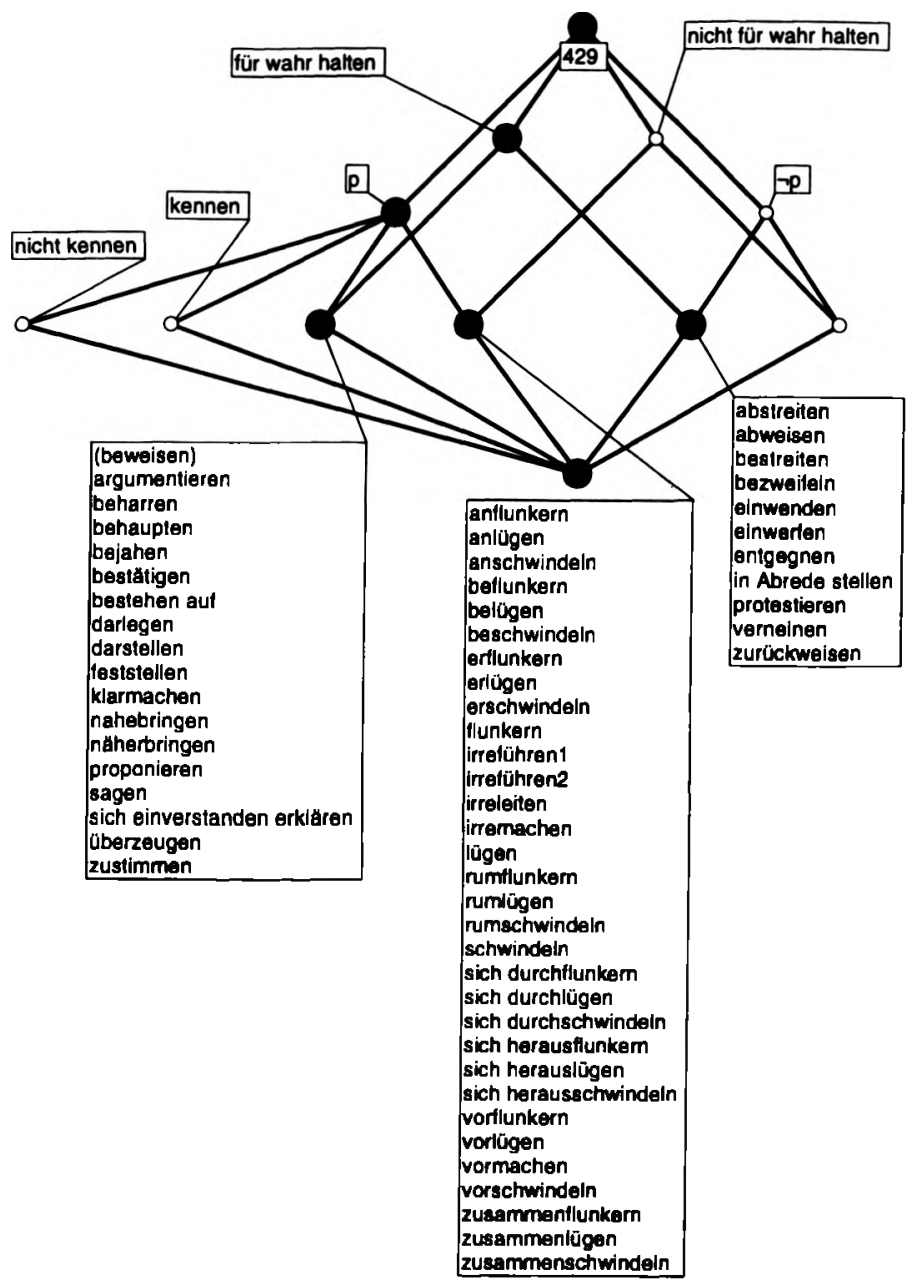

Abbildung 5 Propositionale epistemische Einstellung 
- „handlungsbezogene Einstellung“, d.h. die Intention, $\mathrm{H}$ zu einem Tun zu veranlassen

- „epistemische Einstellung“, d.h. die Intention, H zu einem Glauben zu veranlassen

- „ordinative Einstellung“, d. h. die Intention, H zu einer Einstufung zu veranlassen

- „evaluative Einstellung“, d. h. die Intention, H zu einer Wertung zu veranlassen

- „emotive Einstellung“, d. h. die Intention, $\mathrm{H}$ zu einer Emotion zu veranlassen

- „deklarative Einstellung“, d.h. die Intention, eine institutionelle Tatsache zu schaffen, wie z. B. bei Taufen, Ernennungen, Eröffnungserklärungen u.ả.

Als Beispiel für ein Liniendiagramm ist die intentionale epistemische Einstellung in Abb. 6 wiedergegeben. Hier kann am Diagramm abgelesen werden, daß es 44 Verben mit den Merkmalen "kennen" und "p" gibt und 46 Verben mit den Merkmalen „für wahr halten“ und „p“.

Intentionale epistemische Einstellung

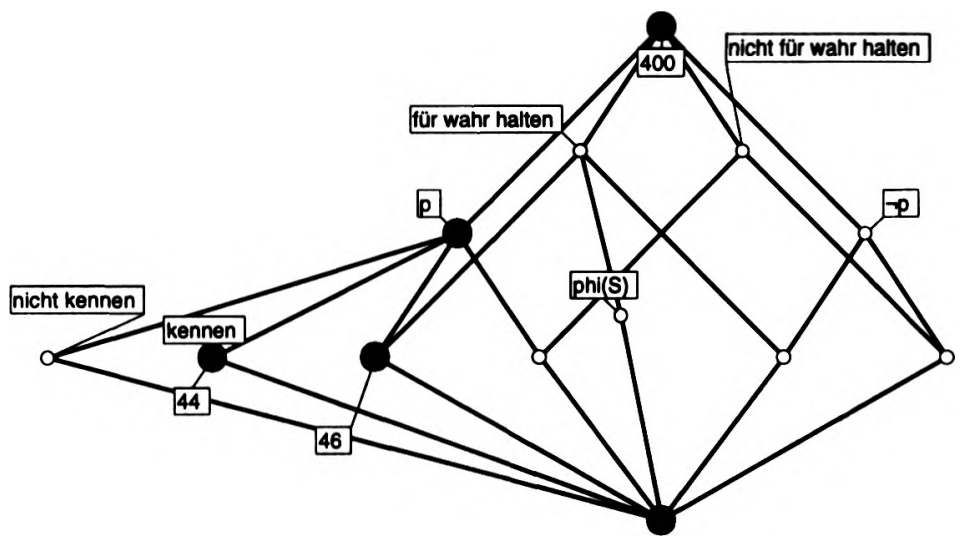

Abbildung 6 Intentionale epistemischeEinstellung

Die interessante Information, die dieses Diagramm liefert, ist, daß es auch zeigt, was es an sprachlichen Möglichkeiten nicht gibt, d.h. die Lücken in der Lexikalisierung des Deutschen, worauf wir in den Beispielen im Abschnitt 4 eingehen.

(3) Die situativen Vorannahmen des Sprechers aus seiner Sicht betreffen: 
- institutionelle Tatsachen wie z. B. Rollenspezifik und Situierung des Gesagten

- Vorannahmen über den Verlauf der Welt und die Annahmen von $\mathrm{H}$, vgl. dazu das Liniendiagramm in Abb. 7

Vorannahmen (S) (primår)

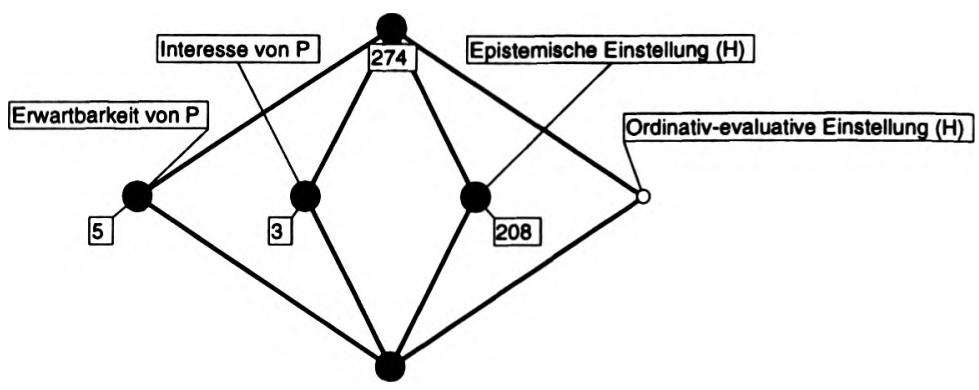

Abbildung 7 Vorannahmen (S)

\section{Möglichkeiten semantischer Erkundungen in TOSCANA}

Die Anwendung von TOSCANA auf die Darstellung der semantischen Struktur bietet für den potentiellen Benutzer zunächst den Vorzug, daß dieser die der Darstellung zugrundegelegte Theorie anschaulich nachvollziehen und damit auch sehen kann, wie die Interpretation der Sprachdaten bezüglich der gewählten Theorie einzuschätzen ist. Für die speziell linguistische Forschung kann die TOSCANA-Anwendung in zwei Weisen genützt werden:

(1) Aufgrund der Möglichkeit der freien Auswahl der einzelnen Themen bzw. Eigenschaften und deren freier Kombination können lexikalische Strukturen „entdeckt" werden, die bisher überhaupt noch nicht in den Blick gekommen sind. 
Ein einfaches Beispiel soll dies verdeutlichen: Wir betrachten zunächst nochmals die Skala der intentionalen epistemischen Einstellung, wie sie in Abb. 6 wiedergegeben ist. Das Liniendiagramm zeigt, daß es 44 Gegenstände (Verben) mit dem Merkmal „kennen p“ und 46 mit dem Merkmal „für wahr

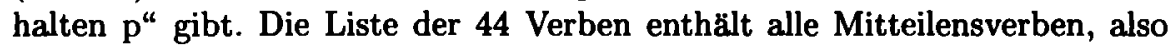
informieren, melden, benachrichtigen, anvertrauen, verlautbaren, verkünden usw. Die Liste der 46 Verben enthält alle Verben, die bezüglich der Sprecherabsicht das Merkmal "S will, daB H p für wahr hält" realisieren, wodurch sich ein Paradigma ergibt, das aus linguistischer Perspektive eher ungewöhnlich ist, nämlich die Kombination von Verben wie: behaupten, beweisen mit Verben wie: lügen, einflüstern, bejahen, beharren auf usw. Für eine Analyse der Versprachlichung von Kommunikationskonzepten ist die Darstellung einer solch ungewöhnlich anmutenden Sicht auf die lexikalische Ordnung der Wörter von nicht zu unterschätzendem heuristischen Wert.

Der Vorteil der Darstellung durch Liniendiagramme liegt nun darin, daß man die zunächst ungewöhnlich zusammengestellte Menge von Verben sukzessive durch die Erkundung von Liniendiagrammen weiterer Skalen differenzierend betrachten kann, indem man sich die Liniendiagramme mehrerer Skalen und deren Beziehungen zueinander in gestuften Liniendiagrammen zeigen läßt (vgl. die Abb. 8, 9, 10 und 11). Für ein gestuftes Liniendiagramm mit zwei Faktoren wählt man zunächst zwei Skalen aus. In einem nächsten Schritt muß man entscheiden, welche der beiden Skalen die Grobstruktur und welche die Feinstruktur darstellen soll - in diesem Sinn sind gestufte Liniendiagramme immer asymmetrisch. Es wird dann zunächst das Diagramm der Grobstruktur gezeichnet, wobei jeder Begriff(sknoten) durch eine Ellipse ersetzt wird. In jede dieser Ellipsen wird eine Kopie der Feinstruktur gezeichnet. Auf diese Weise kann man das direkte Produkt der beiden Skalen vereinfacht darstellen. Die Gegenstands- und Merkmalsnamen werden an die entsprechenden Begriffe der Feinstruktur geschrieben.

Die Leseregeln für gestufte Liniendiagramme sind analog zu denen der einfachen Liniendiagramme. Betrachtet man ein Gegenstand und ein Merkmal, deren Begriffe in der gleichen Ellipse liegen, so gilt das gleiche wie für einfache Liniendiagramme: Der Gegenstand hat das Merkmal, wenn der Merkmalsbegriff vom Gegenstandsbegriff aus durch einen aufsteigenden Streckenzug im Liniendiagramm der Feinstruktur zu erreichen ist. Liegen Gegenstands- und Merkmalsbegriff in zwei verschiedenen Ellipsen, so wird zunächst überprüft, ob die Ellipse des Merkmalsbegriffs durch einen aufsteigenden Linienzug im Liniendiagramm der Grobstruktur von der Ellipse des Gegenstandsbegriffs aus zu erreichen ist. Trifft dies zu, muß zusätzlich noch gelten, daß auch innerhalb eines Diagramms der Feinstruktur der dem Gegenstand entsprechende Knoten durch einen aufsteigenden Linienzug mit dem Knoten, der dem Merkmalbegriff entspricht, verbunden ist. Gelten diese beiden Bedingungen, so hat der Gegenstand das betreffende Merkmal. 
Zur Differenzierung des Paradigmas der Verben wie behaupten, lügen, einflüstern, bejahen, beharren auf usw. betrachten wir das gestufte Liniendiagramm in Abb. 8. Die Skala "Propositionale epistemische Einstellung" stellt die Grobstruktur und die Skala "Intentionale epistemische Einstellung" die Feinstruktur dar. Betrachten wir das Liniendiagramm der Feinstruktur in der Ellipse, die in der Grobstruktur "Propositionale epistemische Einstellung“ den Begriff mit den Merkmalen "nicht für wahr halten" und "p" darstellt. Hier gibt es als einzigen realisierten Begriff der Feinstruktur den Begriff mit den Merkmalen "für wahr halten" und "p". Sie sind nur in der Feinstruktur der obersten Ellipse explizit angegeben, lassen sich aber auf die Feinstrukturen der anderen Ellipsen übertragen. Die Menge der Gegenstände, die den Umfang dieses Begriffs ausmachen, ist die Menge aller Verben, auf die zu-

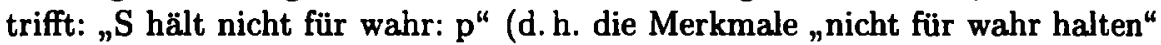
und „p" aus der Grobstruktur, also bezogen auf die propositionale episte-

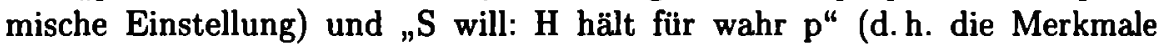
"für wahr halten" und „p" der Feinstruktur, also bezogen auf die intentionale epistemische Einstellung, was auch aus dem Vorsatz ${ }_{n} \mathrm{~S}$ will" deutlich wird), d.h. alle Verben des Lügens. Die Feinstruktur derjenigen Ellipse, die in der Grobstruktur den Begriff mit den Merkmalen „für wahr halten“ und „p“ als Inhalt darstellt, enthält einen realisierten Begriff mit den Merkmalen "für wahr halten“ und "p" (diese Merkmale sind also wieder der Feinstruktur entnommen), der 14 Gegenstände umfaßt: Dies sind alle Verben mit den Merkmalen „S hält für wahr: $p$ " (aus der Grobstruktur, also bezogen auf die propositionale epistemische Einstellung) und ${ }_{n} \mathrm{~S}$ will: $\mathrm{H}$ hält für wahr $\mathrm{p}^{\text {“ }}$ (aus der Feinstruktur, also bezogen auf die intentionale epistemische Einstellung), d.h. alle Behauptens- und Bejahensverben. In dieser Ellipse befindet sich noch ein weiterer realisierter Begriff, nämlich der oberste, das sogenannte Einselement. Der Umfang dieses Begriffes besteht aus all den Verben, die bezüglich der Grobstruktur die Merkmale "für wahr halten" und „p" haben, also die propositionale epistemische Einstellung „S hält p für wahr", jedoch keine Merkmale der Feinstruktur, also keine intentionale epistemische Einstellung. Es dürfte durch dieses Beispiel deutlich geworden sein, in welcher Weise und auch mit welchem heuristischen Nutzen die Erkundungsmöglichkeiten in TOSCANA geeignet sind, den Weg linguistischer Strukturierungen nachvollziehbar und transparent zu machen.

(2) TOSCANA zeigt vermittels der Strukturierung von Begriffsverbänden, die in den Liniendiagrammen anschaulich gemacht sind, jede begrifflich mögliche Position an. Das heißt: alle Kreise, denen keine Gegenstände zugeordnet sind, können potentielle, mögliche Wörter einer Sprache sein. Dies ist ein Ergebnis der Anwendung theoriegeleiteter Skalen, also solcher Skalen, die im vorhinein erstellt wurden, nicht erst in Anbetracht der erhoben Daten. Insofern ist die Anwendung von TOSCANA auf semantisch-konzeptuelle Strukturen eine ideale Ausgangsbasis für kontrastive Untersuchungen des lexikalischen Bestandes verschiedener Spra- 


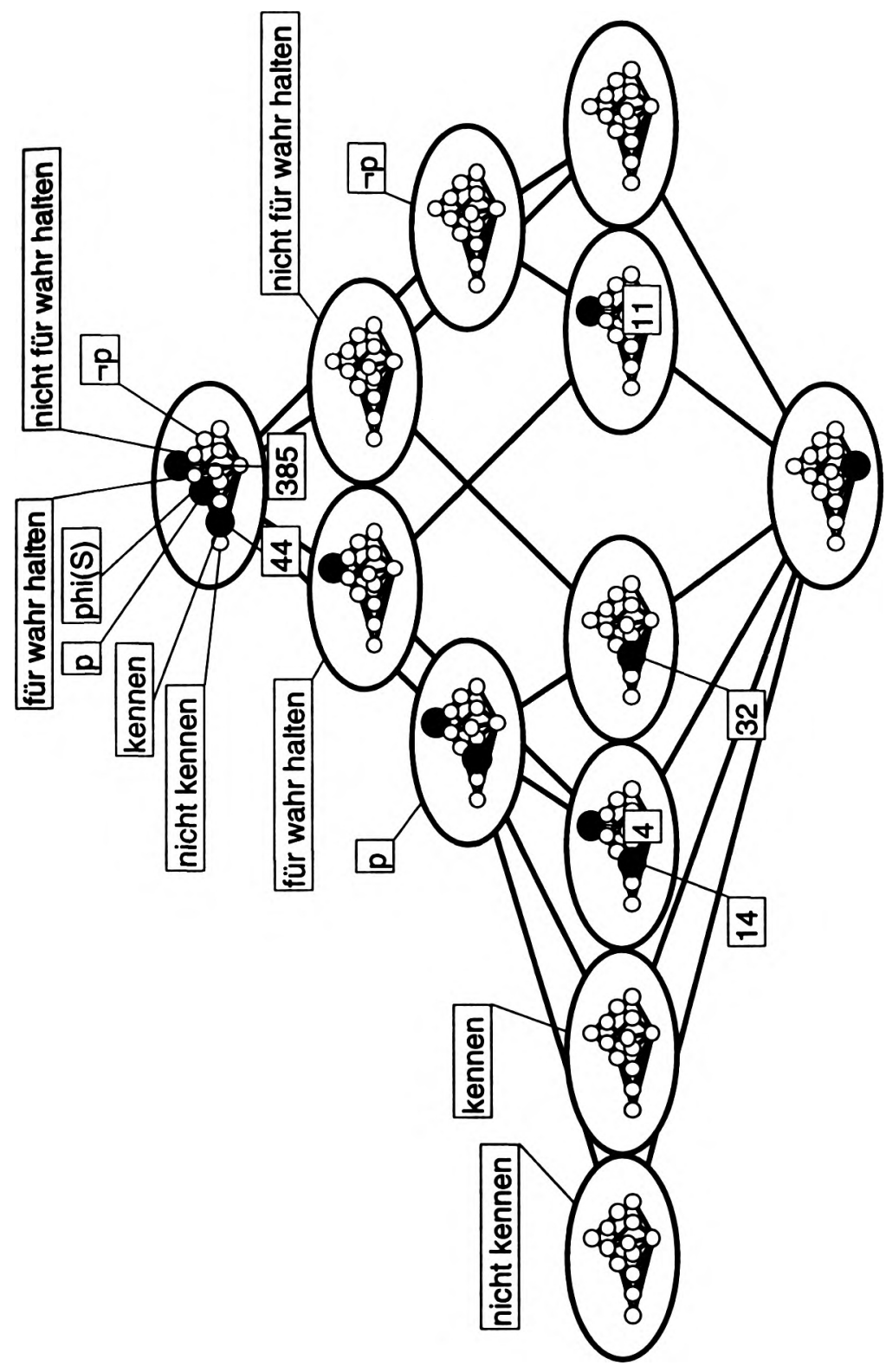

Abbildung 8 Gestuftes Liniendiagramm: Propositionale epistemische Einstellung - Intentionale epistemische Einstellung

chen. Aber auch bezüglich einer Sprache, hier des Deutschen, bietet die Möglichkeit der je individuellen Erkundung des Sprachdschungels interessante Aspekte und Anlaß, darüber nachzudenken, wieso bestimmte kom- 
munikative Konzepte (Merkmalsausprägungen) lexikalisch realisiert sind, andere nicht. Drei Beispiele sollen dies zeigen.

Als erstes Beispiel betrachten wir die Zusammenhänge zwischen propositionaler und intentionaler Einstellung, wie sie im gestuften Liniendiagramm von Abb. 9 dargestellt sind. Die Skala „Propositionale Einstellung“

Propositionale Einstellung

Intentionale Einstellung

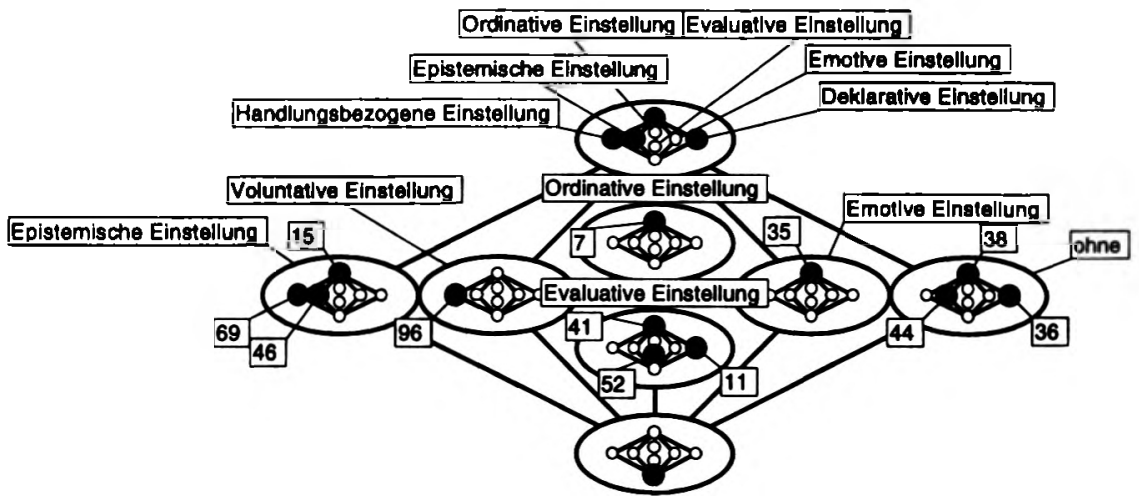

Abbildung 9 Gestuftes Liniendiagramm: Propositionaler Einstellung - intentionale Einstellung

ist als Grobstruktur und die Skala „Intentionale Einstellung“ als Feinstruktur gewählt. Für die Lexikalisierung im Deutschen lassen sich zunächst die folgenden Zusammenhänge erkennen: In der Ellipse zum Merkmalsbegriff „Epistemische Einstellung" der Grobstruktur (Propositionale Einstellung) gibt es zwei realisierte Begriffe, denen auch eine explizite intentionale Einstellung zugeordnet wird. Der eine steht bezüglich der Feinstruktur (Intentionale Einstellung) unter dem Merkmalsbegriff „Handlungsbezogene Einstellung“, der andere unter dem Merkmalsbegriff „Epistemische Einstellung“. Der einzige realisierte Begriff in der Ellipse zum Merkmalsbegriff „Voluntative Einstellung“ der Grobstruktur ist derjenige, der das Merkmal „Handlungsbezogene Einstellung" der Feinstruktur hat. Daran erkennt man, daß aus einer voluntativen propositionalen Einstellung eine handlungsbezogene intentionale Einstellung folgt. Ebenso folgert man, daß ein Verb mit einer evaluativen propositionalen Einstellung eine evaluative intentionale Einstellung hat oder ohne intentionale Einstellung ist. Insgesamt wird die Tendenz deutlich, daß sich die propositionale Einstellung in der intentionalen Einstellung widerspiegelt, d. h. die Verben bezeichnen Bezugssituationen, in denen der Sprecher will, daß der Hörer seine propositionale Einstellung übernimmt. Eine Ausnahme 
bilden allerdings die Zusammenhänge zwischen ordinativer und emotiver propositionaler Einstellung und der intentionalen Einstellung: In beiden Fällen sind die entsprechenden Ellipsen ohne realisierte Begriffe. Für die Lexikalisierung des Deutschen könnte man intuitiv zunächst die folgende Erklärung geben: Für die Verben mit ordinativer, aber ohne evaluative Einstellung gilt, daß der Sprecher seine Setzung oder Einordnung dem Hörer gegebenüber zu erkennen gibt, wobei sich seine Absicht auf eine epistemische Einstellung - nämlich daß der Hörer dies erkennen soll - richtet. Gleiches gilt für den Fall der emotiven Einstellung: Der Sprecher gibt zu erkennen, daß er eine bestimmte Emotion hat, aber er beabsichtigt nicht durch das, was er sagt, zu bewirken, daß der Hörer seine Emotion übernimmt. Gefühle werden ausgedrückt, sie sind aber nicht kommunikativ übertragbar, könnte man etwas salopp formulieren. Ob solche Erklärungsversuche allerdings zutreffend sind, muß eine systematische Analyse, wobei auch andere Merkmalsausprägungen berücksichtigt werden müssen, bei weiteren TOSCANA-Erkundungen erst noch zeigen. Wir hoffen jedoch, daß das Beispiel zeigt, zu welchen Gedanken die Darstellungsweise der Daten anregen kann.

Für unser zweites Beispiel wählen wir die Skala „Mitteilungsgehalt - Fragegehalt" als Grobstruktur und die Skala „Vorannahmen: epistemische Einstellung" als Feinstruktur, vgl. Abb. 10. In der Ellipse zum Merkmalsbegriff "Mitteilungsgehalt" ist fast jeder mögliche Begriff realisiert. Dies bedeutet, daß Verben mit Mitteilungsgehalt Bezugssituationen bezeichnen, in denen der Sprecher alle möglichen Vorannahmen zur epistemischen Einstellung des Hörers haben kann. In der Ellipse zum Merkmalsbegriff „Fragegehalt" gibt es lediglich einen einzigen realisierten Begriff, nämlich „H kennt $\mathrm{p}^{\text {“. Dies mag }}$ auf den ersten Blick trivial erscheinen und den selbstverständllichen Einwand provozieren, daß man jemanden nur dann etwas fragen kann, wenn man annimmt, daß der Hörer die Antwort kennt. Was ist nun aber mit rhetorischen Fragen, Fangfragen, Prüfungsfragen, Suggestivfragen u.ä.? Offensichtlich sind diese Möglichkeiten des Fragens im Deutschen nicht lexikalisiert, d.h. sie können nicht durch ein einfaches Verb bezeichnet werden. Genau dies zeigt uns das gestufte Liniendiagramm auf einen Blick. Es wäre in kontrastiven Untersuchungen zu prüfen, in welchen Sprachen es welche realisierten Begriffe in der Ellipse „Fragegehalt" gibt.

Für unser letztes Beispiel wählen wir die Skala „Mitteilungsgehalt-Fragegehalt" als Grobstruktur und die Skala "Intentionale Einstellung" als Feinstruktur, vgl. Abb. 11. Wie im vorhergehenden Beispiel ist in der Ellipse zum Merkmalsbegriff „Mitteilungsgehalt" der Grobstruktur fast jeder Begriff realisiert: Es gibt also für fast jede Kombination aus „Mitteilungsgehalt" und einer der möglichen intentionalen Einstellungen einen Begriff, der diese Kombination in seinem Inhalt hat. Das bedeutet: Ein Verb mit Mitteilungsgehalt bezeichnet Bezugssituationen, in denen die intentionale Einstellung des Sprechers in keiner Weise festgelegt ist. Anders ist dies bei Verben mit Fragegehalt. Die entsprechende Ellipse enthält nur einen realisierten Begriff. Dieser 


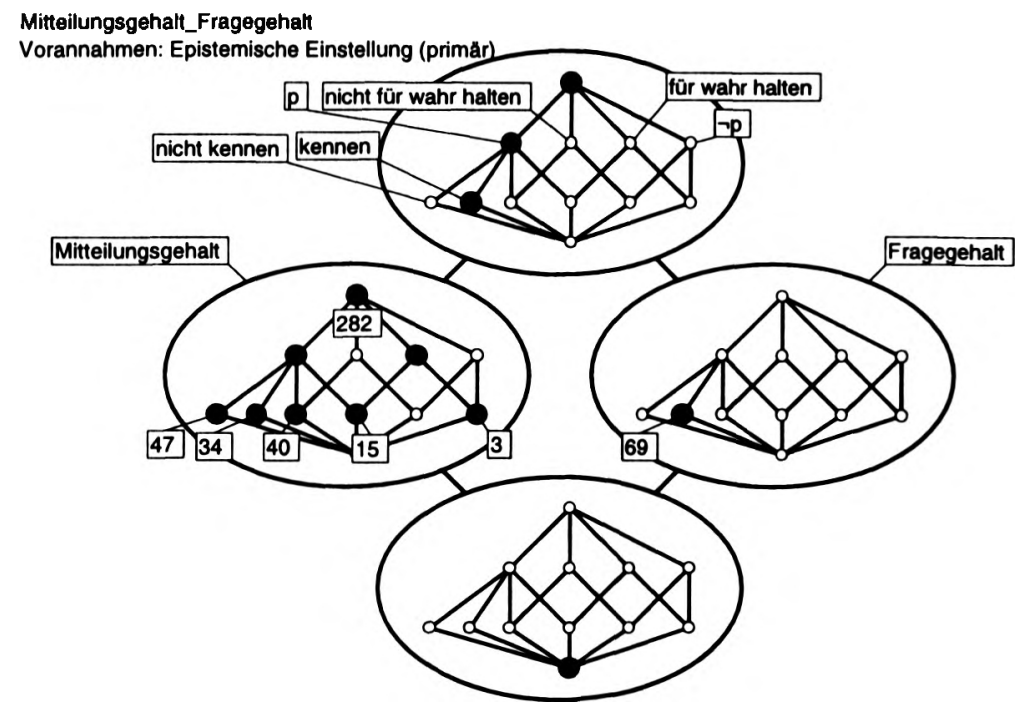

Abbildung 10 Gestuftes Liniendiagramm: Mitteilungsgehalt-Fragegehalt - Vorannahmen: epistemische Einstellung

liegt unter dem Merkmalsbegriff „Handlungsbezogene Einstellung“ der Feinstruktur. Das heißt: für jedes Verb mit Fragegehalt ist die Bezugssituation durch eine handlungsbezogene intentionale Einstellung des Sprechers charakterisiert: Der Sprecher will, daß der Hörer handelt, d.h. antwortet. Auch hier könnte man die gleichen Überlegungen anschließen wie im obigen Beispiel, es sei denn, man stellt sich auf den Standpunkt, Fragen sei ein universales Konzept, das durch die Absicht, eine Antwort zu erhalten, strikt definiert ist.

\section{Ausblick}

Im Unterschied zu den meisten bisher verfügbaren Anwendungen von TOSCANA sind die Daten, die für Sprachanalyse aufbereitet werden müssen, sicher komplexer und vielschichtiger. Es wäre wünschenswert, mehrere Skalen auf einer Stufe miteinander kombinieren zu können, da die hier verwendeten Merkmale teilweise hierarchische Beziehungen zueinander haben. Zum Beispiel sind die Merkmale „epistemische propositionale Einstellung“, „voluntative propostionale Einstellung “, „ordinative propositionale Einstellung“, "ordinative propositionale Einstellung" und „emotive propositionale Einstellung“ Unterklassifikationsmerkmale zum Merkmal „propositionale Einstel- 


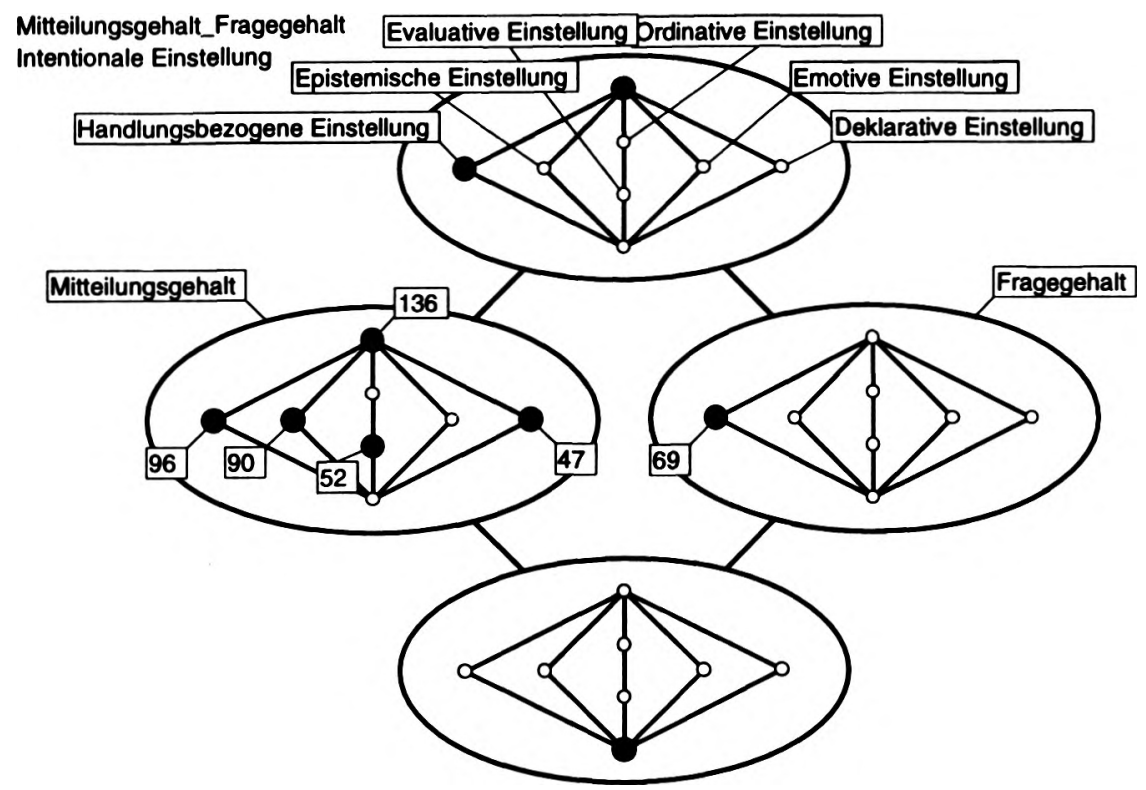

Abbildung 11 Gestuftes Liniendiagramm: Mitteilungsgehalt-Fragegehalt - Intentionale Einstellung

lung“. Die verschiedenen Paradigmen, die durch das Merkmal "propositionale Einstellung" unterschieden werden - also die Verbmengen, die jeweils beim Merkmal „propositionale Einstellung“ die gleiche Ausprägung haben haben durchaus unterschiedliche Struktur. Es ist zum Beispiel sinnvoll, die Verben mit der Ausprägung „epistemische Einstellung“ bezüglich der Skala "epistemische propositionale Einstellung" zu betrachten. Für die Verben mit der Ausprägung „voluntative Einstellung“ ergäbe das jedoch keinen Sinn. Es wäre also wünschenswert, die Skala ,propositionale Einstellung" mit den Skalen ihrer Unterklassifikationsmerkmale dergestalt kombinieren zu können, daß für jeden Begriff der Skala „propositionale Einstellung“ die passende Skala in die Ellipse des entsprechenden Begriffes gezeichnet wird und man so alle Skalen in einem Diagramm zeichnen lassen und somit untersuchen kann. Mit dem Lokalen Skalieren ([St96], [Sc98]) ist es möglich geworden, ein komplexeres Liniendiagramm zu entwickeln, in dem man die verschiedenen Ausprägungen aller Merkmale der propositionalen Einstellung in der Zusammenschau erhalten kann. Das Ergebnis dieser Bemühung geben wir zum Abschluß unseres Beitrags in Abb. 12 wieder. 


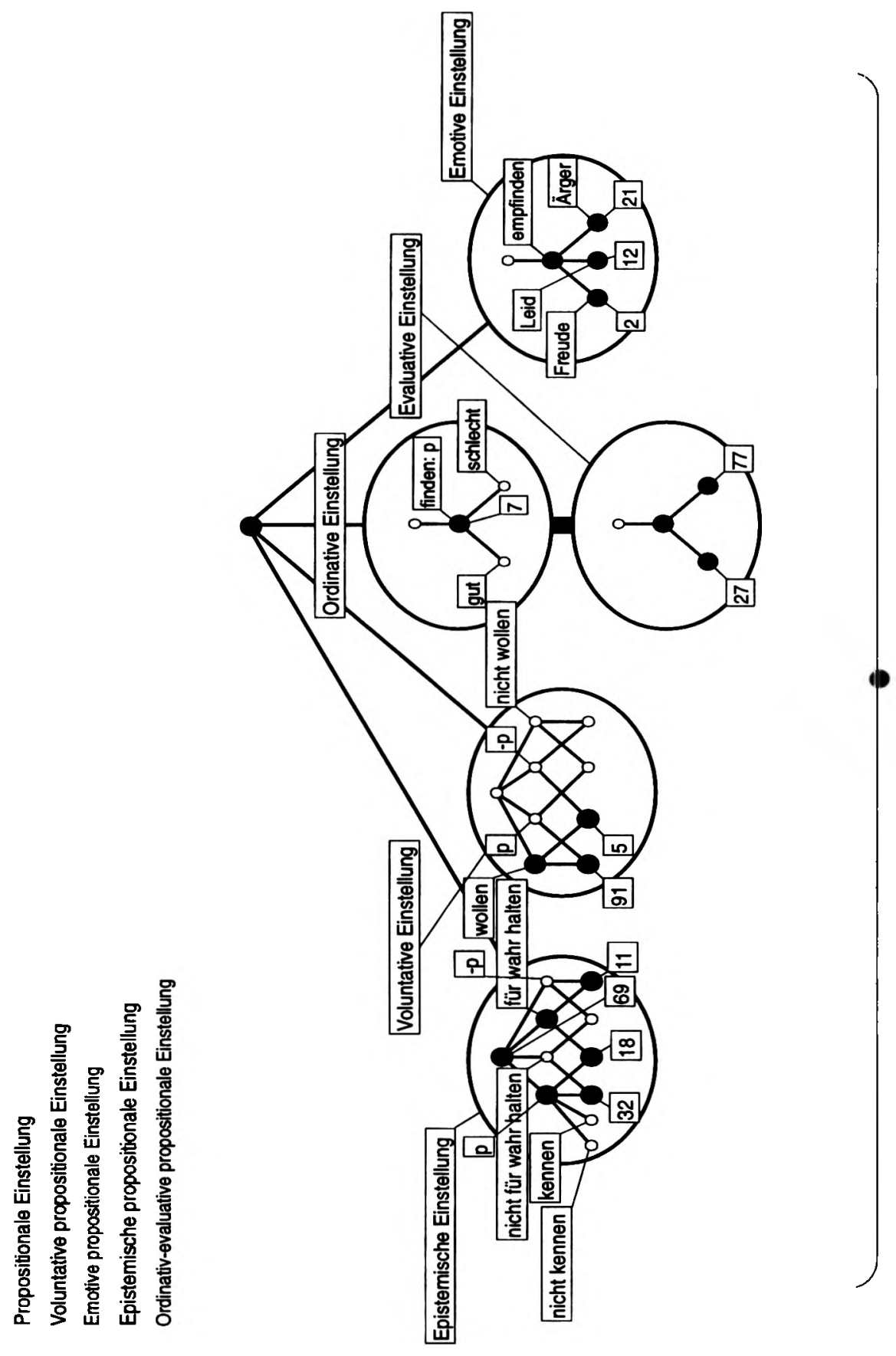

Abbildung 12 Lokales Skalieren 


\section{Literatur}

[Au75] J.L. Austin: How to do things with words. Oxford University Press, Oxford 1975

[Ba79] Th. Ballmer: Probleme der Klassifikation von Sprechakten. In: G. Grewendorf (Hrsg.): Sprechakttheorie und Semantik. Suhrkamp, Frankfurt a. M. 1979, 247-274

[Bau77] K. Baumgärtner: Lexikalische Systeme möglicher Performative. Zeitschrift für germanistische Linguistik,5,3, 257-277

[Bau79] K. Baumgärtner: Lexikalische Systeme möglicher Performative, Manuskript, Stuttgart 1979

[Ha95] G. Harras: Eine Möglichkeit der kontrastive Analyse von Kommunikationsverben. In: H.-P. Kromann und A.L. Kjaer (Hrsg.): Von der Allgegenwart der Lexikographie. Niemeyer, Tübingen 1995, 102-113

[Ha96] G. Harras: sprechen, reden, sagen. Polysemie und Synonymie. In: G. Harras und M. Bierwisch (Hrsg.): Wenn Semantik arbeitet. Klaus Baumgärtner zum 65. Geburtstag. Niemeyer, Tübingen 1996, 191-216

[HaWi94] G. Harras, E. Winkler: A Model for Describing Speech Act Verbs. The Semantic Base of a Polyfunctional Dictionary. In: W. Martin, W. Meijs, M. Moerland, E. ten Pas, E. von Sterkenburg, P. u. P. Vossen (Hrsg.): Euralex 1994, Proceedings, 440-449

[KSVW94] W. Kollewe, M. Skorsky, F. Vogt, R. Wille: TOSCANA - ein Werkzeug zur begrifflichen Analyse und Erkundung von Daten. In: R. Wille und M. Zickwolff (Hrsg.): Begriffliche Wissensverarbeitung: Grundfragen und Aufgaben. B. I.-Wissenschaftsverlag, Mannheim 1994, 267-288

[Me90] G. Meggle: Handlungstheoretische Semantik. Manuskript, Saarbrücken 1990

[Sc98] A. Schatz: Lokales Skalieren: Mathematische Grundlagen und objektorientiertes Design. Diplomarbeit, TU Darmstadt 1998

[Se80] J.R. Searle: Expression and Meaning: Studies in the Theory of Speech Acts. Cambridge University Press, Cambridge 1980

[Se85] J. R. Searle, D. Vanderveken: Foundations of Illocutionary Logic. Cambridge University Press, Cambridge 1985

[St96] G. Stumme: Local scaling in conceptual data systems. In: P.W. Eklund, G. Ellis, G. Mann (Hrsg.): Conceptual structures: Knowledge representation als interlingua. Lecture Notes in Artificial Intelligence 1115. Springer, Heidelberg 1996, 308-320

[U192] M. Ulkan: Zur Klassifikation von Sprechakten. Eine grundlagentheoretische Fallstudie. Niemeyer, Tübingen 1992

[Va90] D. Vanderveken: Meaning and Speech Acts. 2 vols. Cambridge University Press, Cambridge 1990

[Ve80] J. Verschueren: On Speech Act Verbs. Benjamins Publishing Company, Amsterdam 1980

[Wi95] R. Wille: Begriffsdenken: Von der griechischen Philosophie bis zur Künstlichen Intelligenz heute. In: Dilthey-Kastanie. Ludwig-GeorgsGymnasium, Darmstadt 1995, 77-109

[Win96] E. Winkler: Kommunikationskonzepte und Kommunikationsverben. In: J. Grabowski, G. Harras, T. Herrmann, (Hrsg.): Bedeutung. Konzepte. Bedeutungskonzepte. Theorie und Anwendung in Linguistik und Psychologie. Westdeutscher Verlag, Opladen 1996, 256-276 